\title{
Mechanical properties of hierarchical lattices
}

\author{
Andrea Vigliotti, Damiano Pasini* \\ Department of Mechanical Engineering - McGill University 817 Sherbrooke Street West, \\ H3A 0C3, Montreal, QC, CANADA
}

\begin{abstract}
This paper focuses on the stiffness and strength of lattices with multiple hierarchical levels. We examine two-dimensional and three-dimensional lattices with up to three levels of structural hierarchy. At each level, the topology and the orientation of the lattice are prescribed, while the relative density is varied over a defined range. The properties of selected hierarchical lattices are obtained via a multiscale approach applied iteratively at each hierarchical level. The results help to quantify the effect that multiple orders of structural hierarchy produces on stretching and bending dominated lattices. Material charts for the macroscopic stiffness and strength illustrate how the property range of the lattices can expand as subsequent levels of hierarchy are added. The charts help to gain insight into the structural benefit that multiple hierarchies can impart to the macroscopic performance of a lattice.
\end{abstract}

Keywords: lattice materials, periodic cellular materials, multiscale mechanics, stiffness and strength properties, material property charts

\section{Introduction}

Materials with a hierarchical microstructure are very common in Nature and are a remarkable source of inspiration for the development of new materials. Wood at the macroscopic level, for instance, can be loosely described as made of an arrangement of hollow tubes, whose walls have a microstructure of hemicellulose reinforced with lignin [8]. In bone, up to seven orders of hierarchical organization can be identified, each with a defined structural

\footnotetext{
*Corresponding author

Email address: damiano.pasini@mcgill.ca (Damiano Pasini)
} 
architecture. At the larger length scale we have the trabeculae, which make up the cancellous bone; the trabeculae are made of a network of osteons, which in turn are made of porous hollow fibres, each consisting of protein fibrils $[21,30]$. It is proven that nesting multiple hierarchical levels confers significant benefits to the mechanical properties of biological materials $[2,12,15,31,10]$. Structural hierarchy in biological materials is the result of a lengthy optimization process, through which the material is constantly prompted by the natural environment to simultaneously fulfil a broad range of multifunctional and conflicting requirements $[12,6]$. In wood, the cellular tissue permits the circulation of vital fluids, and confers high compliance and strength to each organ of the plant. The trabecular structure of bones allows the continuous regeneration and maintenance of the structure, while bearing the operational loads. Nacre, the material of seashells and turtle shells, is made of a complex multi-layered arrangement of calcium carbonate tablets, submerged in a soft organic matrix. It has been demonstrated that the exceptional toughness of Nacre, which far exceeds that of its constituents, is controlled by the architecture of its microstructure $[1,5]$. The high toughness of Nacre is crucial to protect the soft organisms enclosed in the shell, and to allow the growth of the shield.

Whereas environmental constraints guide the adaptive process of material formation over millions of years, engineers can resort to additive manufacturing and nanotechnology to build - in a fairly short time frame and at affordable cost - advanced materials with multiple orders of microstructural organization $[1,8]$. The latest advance in additive manufacturing has driven recent research to the understanding of the properties of hierarchical materials $[32,23,9,17,19]$. The concept of structural hierarchy has been exploited in engineering for a long time, one notable example being the Eiffel tower, a third order hierarchical structure whose relative density, the ratio between the volume occupied by the structure and the volume occupied by the solid material, is just $1.2 \times 10^{-3}$ [14]. In the literature, a seminal work on materials with structural hierarchy is the one by Lakes, who examined a set of natural and artificial hierarchical materials. In this work, Lakes first proposed a compact expression for the stiffness and strength of materials with isotropic structure at each hierarchical level. Parkhouse also showed that the process of sub-structuring can be recursively applied to each element of a macrostructure, thus no clear distinction exists, in principle, between structure and material [16]. The effect of material heterogeneity, which occurs by structuring a material at multiple length scale with properties that are 
dissimilar from one order of scale to another, was also studied by Yao et al. [33]. In an experimental and numerical work on the cortical bone of a bovine, Yao et al. illustrated the benefit that structural hierarchy generates in reducing stress concentration at the nanoscale, as well in improving strength and energy dissipation. Sen and Buehler [22] showed that structural hierarchy enhances toughness and resistance to crack propagation in brittle materials without the need to introduce additional materials. More recently, Fleck et al. [6] suggested that materials with multiple orders of structural hierarchy have the potential to further improve the performance of lattice materials, in particular to yield higher stiffness, strength and fracture toughness at lower density. Sandwich panels with hierarchical cores have been also the object of recent investigations $[28,13]$. From these works, it emerges that for a given density the strength of a panel with two levels of hierarchy in its core can be up to 12.5 times higher than the strength of a panel with a core with a single hierarchical order. Another example is the work of Zhao et al. [34], who designed, manufactured and tested a hierarchical woven lattice composite. The lattice walls were made of a woven textile sandwich composite, and at the highest level, three lattice topologies were considered: the square, the triangular and the Kagome lattice. It was shown that the presence of a level of hierarchy in the lattice elements significantly enhances the capacity of the lattice to absorb energy. In a more recent study, Torrents et al. [25] manufactured and tested a nickel-based microlattice materials with three orders of structural hierarchy from the nanometer to the millimeter scale, and relative density in the range $1 \times 10^{-4}-8.5 \times 10^{-1}$. A macroscopic stiffness and strength of one order of magnitude larger than those of existing materials were observed in the lowest relative density range, and were attributed to the existence of multiple hierarchical levels. In another recent work, RayneauKirkhope et al. [20] applied fractal theory to design beams with multiple levels of hierarchy, thereby obtaining improved buckling strength to mass ratio. The authors also manufactured a beam with two levels of hierarchy by means of rapid prototyping to validate the theoretical results.

In this paper, we use a multiscale approach to quantify the effect of multiple structural hierarchies on a material with a lattice architecture. We show that by nesting multiple levels of lattice hierarchies, and by varying the relative density at each level, the property design space of the solid material can be expanded to reach unexplored areas of the material charts. In the first part of the paper, we examine the stiffness and strength of four planar lattices with high relative density. As expected, when the relative density 
of all levels tends to unity, the overall properties converge to those of the solid material. The results show that the lattice topology has a strong impact on the overall properties of the material. Bending dominated lattices tend to gain more benefit from the existence of multiple hierarchies, thereby increasing significantly the specific stiffness. Stretching dominated lattices, on the other hand, have already an optimal configuration with respect to stiffness, and thus do not show a major improvement. With respect to plastic yielding, a detriment of the overall material strength is observed in high density lattices due to the recursive effect of stress concentration that occurs at each hierarchical level. In the second part of the paper, we analyse four three-dimensional lattices with both open and closed cells, and examine the stiffness and buckling strength of the material.

\section{The multiscale scheme}

As an example of a hierarchical lattice, consider the planar structure shown in Figure 1. At the topmost level, $h-3$, the lattice has a hexagonal topology and the material of the struts is made of a Kagome lattice, which is the hierarchical level 2. The struts of the lattice at level 2 hold another level of substructure, where the material consists of a square lattice. At the level 1 of the hierarchy, the lattice is made of a uniform solid material, level 0 . In this paper, we are interested in describing how the properties of the

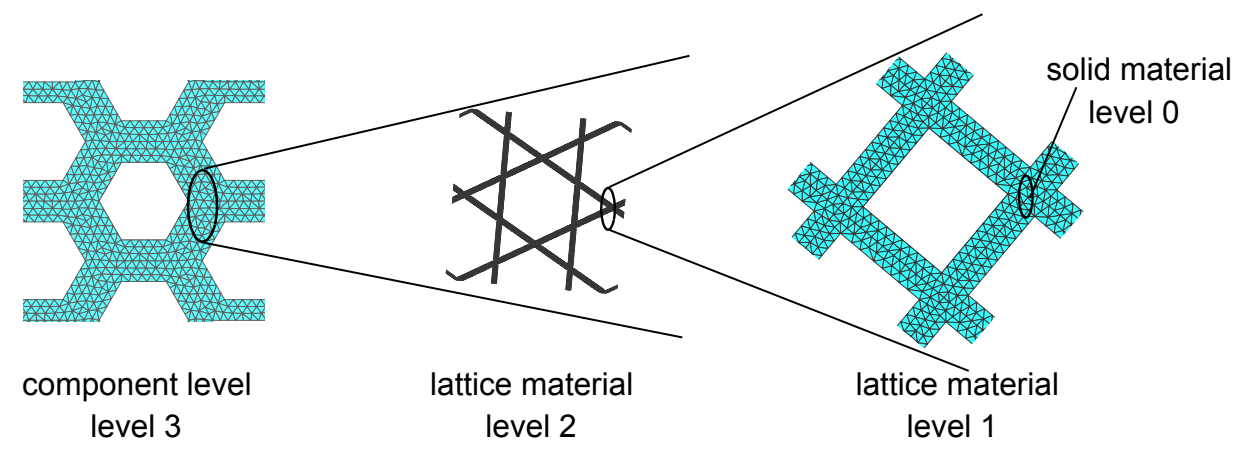

Figure 1: Microlattice with multiple hierarchical levels

lattice at the top level change if the number of hierarchical orders and geometrical parameters of the lattices vary at each level. While the relative density of a hierarchical lattice is simply the product of the relative densities at each level, this does not hold for stiffness, strength and other properties. 
For example, the stiffness of the lattice at a given level is governed by the lattice topology, the geometrical parameters of the unit cell at that level, and the properties of the solid material. Only if we prescribe an isotropic solid material and an isotropic topology at each order, the resulting lattice displays isotropic macroscopic properties. In this case, we can resort to the compact expressions proposed by Lakes [14] for the Young's modulus and the strength. However in the general case, when the parameters of the lattice at each level are dissimilar, it is necessary to follow a bottom up approach starting from the solid material level, and derive the properties of the lattice at each level from the parameters of the material at the former level.

It is well known that the macroscopic properties of a lattice material are governed by the properties of its microarchitecture, in particular by the geometry of the unit cell. In principle, a direct numerical approach, which would involve the individual modelling of each lattice edge or wall, might be possible. Yet, a detailed model of the lattice becomes unfeasible if the length scale of the lattice is negligible with respect to the length scale of the component. In these cases, a viable strategy is to resort to a homogenization approach to calculate the macroscopic properties of the lattice, and then model the component as a homogeneous medium. In previous papers $[26,27]$, the authors presented a multiscale homogenization method that allows determine the mechanical properties of a lattice material. Given a finite element model of the unit cell, this method enables to calculate the macroscopic stiffness tensor of the lattice, and the internal forces in the lattice elements to assess the lattice strength. This method is iteratively applied in this paper to calculate the mechanical properties of lattices with multiple levels of hierarchy.

We assume that the microscopic stiffness of the material at each level is given by the macroscopic stiffness of the lattice at the former level. As illustrated in Figure 3, given the material stiffness, $\mathbf{K}_{\mu_{i}}$, and the geometry of the unit cell, we first find the stiffness matrix of the unconstrained unit cell, $\mathbf{K}_{u c_{1}}$. Then, by applying proper periodic boundary conditions on the unit cell, we calculate the macroscopic stiffness of the lattice at that level, $\mathbf{K}_{M_{i}}$. Starting from the first level, where the lattice is made of the solid material $\left(\mathbf{K}_{\mu_{1}}=\mathbf{K}_{s}\right)$, we evaluate the macroscopic lattice stiffness tensor, $\mathbf{K}_{M_{1}}$, at level 1, which represents the microscopic stiffness of the material at level 2, $\left(\mathbf{K}_{\mu_{2}}=\mathbf{K}_{M_{1}}\right) . \quad \mathbf{K}_{\mu_{2}}$ is then used to obtain the macroscopic stiffness of the lattice at the second level. This approach can be repeated recursively to calculate the macroscopic stiffness of the material at the topmost level. 


\begin{tabular}{|c|c|c|c|c|}
\hline First level & Second level & & $(n-1)^{t h}$ - level & $n^{t h}-$ level \\
\hline $\begin{array}{l}K_{\mu_{1}}=K_{s} \\
K_{u c_{1}} \\
K_{\mathrm{M}_{1}}\end{array}$ & $\begin{array}{l}K_{\mu_{2}}=K_{\mathrm{M}_{1}} \\
K_{u c_{2}} \\
K_{\mathrm{M}_{2}}\end{array}$ & - - & $\begin{array}{l}K_{\mu_{n-1}}=K_{\mathrm{M}_{2}} \\
K_{u c_{n-1}} \\
K_{\mathrm{M}_{\mathrm{n}-1}}\end{array}$ & $\begin{array}{l}K_{\mu_{n}}=K_{\mathrm{M}_{\mathrm{n}-1}} \\
K_{u c_{n}} \\
K_{\mathrm{M}_{\mathrm{n}}}\end{array}$ \\
\hline
\end{tabular}

Figure 2: Schematic protocol to recursively calculate the material stiffness of a hierarchical material. B.C.: boundary conditions.

To obtain the strength of the material, we follow the reverse path. As shown in Figure 3, starting from the topmost level, where macroscopic boundary conditions are applied, we solve the structural problem by assuming the domain as a continuum with stiffness $\mathbf{K}_{M_{n}}$, and we find the strain distribution at the $n^{\text {th }}$ level. Before examining the material at the $(n-1)^{t h}$ hierarchical level, we first verify that macroscopic buckling does not occur at the $n^{t h}$ level. Then, let $\boldsymbol{\epsilon}_{\mu_{n}}$ be the components of the strain field in the most stressed location, we solve the microscopic problem at level $(n-1)^{t h}$ considering $\boldsymbol{\epsilon}_{M_{n-1}}=\boldsymbol{\epsilon}_{\mu_{n}}$ as the macroscopic boundary conditions applied to the lattice at the $(n-1)^{t h}$ level. The procedure is iteratively applied until we reach the first hierarchical level, where the lattice is made of an unstructured material. Here, the effective stress in the solid material is calculated to verify whether plastic yield occurs.

\begin{tabular}{|c|c|c|c|c|}
\hline$n^{t h}$-level & $(n-1)^{t h}$ - level & & Second level & First level \\
\hline Macro B.C. & $\epsilon_{M_{n-1}}=\epsilon_{\mu_{n}}$ & & $\epsilon_{M_{2}}=\epsilon_{\mu_{3}}$ & $\epsilon_{M_{1}}=\epsilon_{\mu_{2}}$ \\
\hline$u_{M_{n}}$ & $u_{M_{n-1}}$ & " & $u_{M_{2}}$ & $u_{M_{1}}$ \\
\hline$\epsilon_{\mu_{n}}$ & $\epsilon_{\mu_{n-1}}$ & & $\epsilon_{\mu_{2}}$ & $\epsilon_{S}, \sigma_{s}$ \\
\hline
\end{tabular}

Figure 3: Schematic protocol to recursively calculate the material strength of a hierarchical material

We note that the procedure introduced in this paper is general; it can be applied to any hierarchical structure with any arbitrary topology and orientation of the lattice cell at each level. Here, we focus on hierarchical lattices in which the topology and orientation of the cells do not change among levels, and at each level we vary the relative density at each order of the structural hierarchy.

We remark that the results obtained in this paper are valid under the 
assumption that the lattice remains periodic after the deformation. Thus, the findings are strictly valid for an infinite lattice subjected to a uniform field of macroscopic deformation. Following an engineering approach, the results can be considered accurate as long as the length scale of the lattice is negligible with respect to the length scale of the macroscopic strain. Recent numerical investigations $[18,7]$ on discrete lattices showed that a boundary layer regions forms in proximity of localised deformation, such as in the neighbourhood of cracks, domain boundaries and where external loads are applied. The extent of this region depends on the topology of the lattice. For example, for triangulated and hexagonal lattice, the boundary region is limited to a few strut lengths; for the Kagome, it can be up to 10 cells. The lattice deformation returns then uniform in the material domain outside the boundary layer. On the base of this observation, we can conclude that at least one order of magnitude should separate the length scale of two levels in the structural hierarchy. We note that the effects of length scale separation among hierarchical orders is not considered in this paper; thus the results are valid in the hypothesis of asymptotic separation. Yet, the findings provide insight into the effect of multiple hierarchical levels on the stress distribution, and into the existence of stress concentration in hierarchical lattices.

\section{Analysis of high density two-dimensional lattices}

If the unit cell is modelled with Euler Bernoulli beams and the solid material is isotropic, closed-form expressions for the macroscopic stiffness and the internal forces in the lattice elements can be obtained as a function of the slenderness of the edges and the Young's modulus of the solid material. Table 1 reports the stiffness tensor for the lattices examined in this section [26]. For comparison purposes, the stiffness tensor of an isotropic material is also included. We remark that the expressions in Table 1 are valid under the hypothesis that the edges behave as Euler Bernoulli beams; this applies to low density lattice with slenderness ratio $\lambda<1 / 20$. We also observe that the homogenization method used to derive the expressions in Table 1 does not retain any information about the actual length scale of the lattice; in fact all the expressions are solely function of the Young's modulus of the solid material and of the slenderness of the beams; hence, the length scale of the lattice does not appear in the expressions.

An important criterion to classify lattice materials is the mechanism of deformation of the unit cell members. In stretching dominated lattices, any ex- 


\begin{tabular}{|c|c|}
\hline Kagome & Hexagonal \\
\hline $\begin{array}{c}\mathbf{K}=\frac{\sqrt{3} E_{s} \lambda}{16}\left[\begin{array}{ccc}\lambda^{2}+6 & 2-\lambda^{2} & 0 \\
2-\lambda^{2} & \lambda^{2}+6 & 0 \\
0 & 0 & \lambda^{2}+6\end{array}\right] \\
\rho^{*}=\sqrt{3} \lambda \\
\mu_{1}=\frac{\sqrt{3} E_{s} \lambda}{2} \\
\mu_{2}=\frac{\sqrt{3} E_{s} \lambda}{8}\left(\lambda^{2}+2\right) \\
\mu_{3}=\frac{\sqrt{3} E_{s} \lambda}{8}\left(\lambda^{2}+2\right)\end{array}$ & $\begin{array}{c}\mathbf{K}=\frac{E_{s} \lambda}{2 \sqrt{3}\left(\lambda^{2}+1\right)}\left[\begin{array}{ccc}3 \lambda^{2}+1 & 1-\lambda^{2} & 0 \\
1-\lambda^{2} & 3 \lambda^{2}+1 & 0 \\
0 & 0 & 2 \lambda^{2}\end{array}\right] \\
\rho^{*}=\frac{2}{\sqrt{3}} \lambda \\
\mu_{1}=\frac{E_{s} \lambda}{\sqrt{3}} \\
\mu_{2}=\frac{2}{\sqrt{3}} \frac{E_{s} \lambda^{3}}{\lambda^{2}+1} \\
\mu_{3}=\frac{1}{\sqrt{3}} \frac{E_{s} \lambda^{3}}{\lambda^{2}+1}\end{array}$ \\
\hline $\begin{array}{c}\text { Triangular } \\
\mathbf{K}=\frac{\sqrt{3} E_{s} \lambda}{4}\left[\begin{array}{ccc}\lambda^{2}+3 & 1-\lambda^{2} & 0 \\
1-\lambda^{2} & \lambda^{2}+3 & 0 \\
0 & 0 & \lambda^{2}+1\end{array}\right] \\
\rho^{*}=2 \sqrt{3} \lambda \\
\mu_{1}=\sqrt{3} E_{s} \lambda \\
\mu_{2}=\frac{\sqrt{3} E_{s} \lambda}{2}\left(\lambda^{2}+2\right) \\
\mu_{3}=\frac{\sqrt{3} E_{s} \lambda}{4}\left(\lambda^{2}+2\right)\end{array}$ & $\begin{array}{c}\text { Square } \\
\mathbf{K}=E_{s} \lambda\left[\begin{array}{lll}1 & 0 & 0 \\
0 & 1 & 0 \\
0 & 0 & \frac{\text { lambda }}{2}\end{array}\right] \\
\rho^{*}=2 \lambda \\
\mu_{1}=E_{s} \lambda \\
\mu_{2}=E_{s} \lambda \\
\mu_{3}=\frac{E_{s} \lambda}{2}\end{array}$ \\
\hline $\begin{array}{c}\text { Isotropic material } \\
\begin{array}{c}\mathbf{K}=\frac{E_{s} \lambda}{1-\nu^{2}}\left[\begin{array}{ccc}1 & \nu & 0 \\
\nu & 1 & 0 \\
0 & 0 & \frac{1-\nu}{2}\end{array}\right] \\
\rho^{*}=1 \\
\mu_{1}=\frac{E_{s}}{1-\nu} \\
\mu_{2}=\frac{E_{s}}{1+\nu} \\
\mu_{3}=\frac{E_{s}}{2(1+\nu)}\end{array}\end{array}$ & \\
\hline
\end{tabular}

Table 1: Properties of selected low density two-dimensional lattices. In the above expressions, $\lambda=t / L$ is the slenderness ratio, where $t$ is the edge thickness and L is the length. $E_{s}$ is the Young's modulus of the solid material and $\nu$ the Poisson's ratio. $\mathbf{K}$ is the macroscopic stiffness tensor of the lattice that yields $\boldsymbol{\sigma}=\mathbf{K} \boldsymbol{\epsilon}$, with $\boldsymbol{\epsilon}=\left[\epsilon_{x}, \epsilon_{y}, \gamma_{x y}\right]$, and $\boldsymbol{\sigma}=\left[\sigma_{x}, \sigma_{y}, \tau_{x y}\right] . \rho^{*}$ is the relative density, $\mu_{i}$ are the eigenvalues of the stiffness tensor of the lattice. The expressions are valid for $\lambda<1 / 20$. For comparative purposes, the corresponding expressions are also given for a uniform isotropic material.

ternal load generates axial forces in the lattice elements, as opposed to what occurs in bending dominated lattices, where lattice edges deform mainly by bending. Table 1 reports the stiffness matrices of the lattices analysed in this paper: the Kagome and triangular, which are stretching dominated lattices, and the square and hexagonal lattices, bending dominated. Deshpande 
et al. [3] showed that the deformation mode of a lattice depends on the nodal connectivity and on the determinacy of the pin-jointed version of the lattice. If the pin-jointed lattice is kinematically indeterminate, i.e. if it holds any zero-energy deformation mode, then its rigid-joint version responds mainly by bending. In general, a lattice is more compliant for the modes that produce bending in its element, and stiffer for the modes that are withstood by axial force.

A suitable mean to compare the stiffness properties of lattices is to consider the eigenspace of the matrix form of their stiffness tensor. The largest eigenvalue corresponds to the deformation mode for which the lattice offers the highest stiffness; the smallest eigenvalue refers to the deformation mode for which the lattice is most compliant. Thus the eigenvalues of the lattice stiffness matrix represent the actual bounds of the lattice stiffness. For the lattices listed in Table 1, including the solid isotropic material, the eigenvectors are given by

$$
\boldsymbol{\epsilon}_{1}=[1,1,0] \quad \boldsymbol{\epsilon}_{2}=[1,-1,0] \quad \boldsymbol{\epsilon}_{3}=[0,0,1]
$$

where the components of the strain are $\boldsymbol{\epsilon}_{i}=\left[\epsilon_{x x}, \epsilon_{y y}, \gamma_{x y}\right], \boldsymbol{\epsilon}_{1}$ corresponds to equibiaxial strain, $\boldsymbol{\epsilon}_{2}$ corresponds to alternate tension-compression, and $\boldsymbol{\epsilon}_{3}$ corresponds to pure shear. For the lattices analysed in this paper, the eigenvalues corresponding to each eigenvector are listed in Table 1. As we can observe, the highest eigenvalue corresponds to the equibiaxial conditions, whereas the lowest corresponds to the pure shear. Since any arbitrary macroscopic stress state can be decomposed as the sum of the eigenvectors of the lattice stiffness matrix, it follows that the lattice will exhibit the largest deformation. Eventually, the lattice would fail, as determined by the mode corresponding to the smallest eigenvalue, for which the lattice is the most compliant. Thus, we can consider the stiffness and the strength of a lattice be governed by the smallest eigenvalue of the stiffness matrix. We also observe that the expressions of the eigenvalues (Table 1) follow dissimilar scaling laws. For stretching dominated lattices, all modes produce mainly axial forces, and all the eigenvalues scale with the first power of $\lambda$. On the other hand for bending dominated lattices, the largest eigenvalue corresponding to equibiaxial strain, for which the lattices responds by axial forces, scales with $\lambda$. The smallest eigenvalue, corresponding to shear, for which the lattice responds with the bending of the edges, scales with $\lambda^{3}$. In general, for stretching dominated modes $\mu_{i}$ scales with $\lambda$, because the stiffness is governed by the cross section area. For bending dominated modes, $\mu_{i}$ scales 
with $\lambda^{3}$, as the stiffness is controlled by the second moment of area of the cross section. It follows that reducing the relative density, and thus $\lambda$, by a factor of 10, decreases the stretching eigenvalues by a factor of 10, and the bending eigenvalues by a factor of 1000 .

For high density lattices, the stubby elements of the unit cell should be modelled with continuous plane stress elements. This choice enables not only to account for the actual stress distribution in the edges if the hypothesis of slenderness is not met, but also to accurately determine the joint stiffness and relative density. In contrast in a discrete model, the cross-section properties are assumed to be concentrated in the centre of the element cross-section, and overlapping volumes at the joints are not correctly accounted. Figure 4 shows the finite element meshes of the topologies under investigation, for three alternative values of relative density from high to low. In dark blue is shown the unit cell used to tessellate the plane.

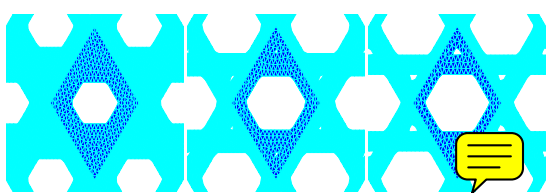

(a) Kagome lattice

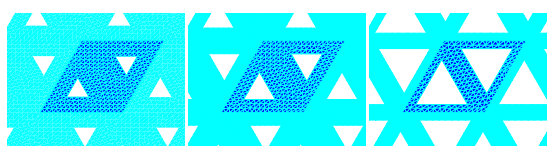

(c) Triangular lattice

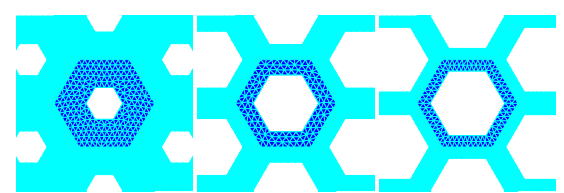

(b) Hexagonal lattice

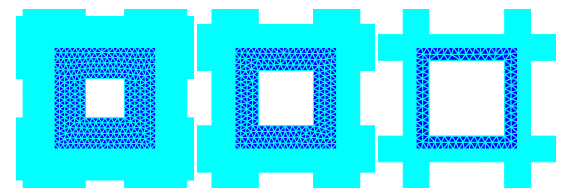

(d) Square lattice

Figure 4: FE meshes of selected two-dimensional lattices for decreasing values $\rho^{*}$

Figure 5 shows the stiffness maps for lattices with one, two and three levels of hierarchy. For each lattice, we plot the highest and the lowest eigenvalue of the stiffness matrix, normalised by the Young's modulus of the solid material. The stiffness of the lattice with one hierarchical level, made of the solid material, is shown by a continuous line, while the symbols refer to the properties of the lattices with nested levels of structural hierarchy. As can be observed, as the relative density approaches one, the stiffness of all lattices approaches that of the solid material, i.e. the largest eigenvalue approaches the equibiaxial stiffness eigenvalue, $\mu_{1}=E_{s} /(1-\nu)$, and the smallest eigen- 
value approaches the shear stiffness eigenvalue $\mu_{3}=E_{s} /(2(1+\nu))$, where $\nu=0.3$. With reference to the lattices with one hierarchical level, we also observe a substantial difference between stretching dominated and bending dominated lattices. For the former, the equibiaxial and the shear stiffness scale with the same power law. Thus no deformation mode prevails over the other as the relative density decreases. Bending dominated lattices, on the other hand, tend to be more compliant under shear loading as $\rho^{*}$ tends to zero. We remark that due to the symmetries of the lattices here considered, an equibiaxial load produces only normal stress in both the stretching and the bending dominated topologies. In contrast, a shear macroscopic load produces essentially bending in the edges of the square and of the hexagonal lattices, whereas it generates axial forces in the elements of the triangular and the Kagome lattices. This explains why the shear and the equibiaxial eigenvalue of stretching dominated lattice follow the same scaling law, and for bending dominated the lattice tends to be significantly more compliant as the relative density decreases. These observation are consistent with the stiffness expressions of low density lattices, obtained with discrete structural elements and reported in Table 2.

Let us now consider the properties of lattices with two and three hierarchical levels. In Figure 5, the points relative to lattices with two hierarchical levels are shown with an empty square marker, while the points relative to three hierarchical level lattices are shown with a solid diamond. As can be seen, the existence of more than one hierarchical level enables to remarkably extend the design space of the lattice. Lattices with one hierarchical level are restricted to a curve segment on the property space, whereas lattices with multiple hierarchical levels can span over a wider region of the material property space. Furthermore, Figure 5 illustrates that nesting multiple hierarchical levels produces dissimilar effects in stretching and bending dominated modes. For the former, the points representing lattices with two and three hierarchical levels are located below the solid line. On the other hand, for the latter the points relative to lattices with higher hierarchy are located above the line representing the stiffness of plain lattices in solid material. From this observation, we conclude that the shear stiffness (represented by the minimum eigenvalue in Figure 5) of the hexagonal and square lattices benefits from the presence of structural hierarchy. In fact, for a given relative density, configurations with multiple hierarchy produce stiffer lattices. In addition, Figure 5 shows how the properties evolve with the number of hierarchical levels. An increase in the number of hierarchical levels is beneficial to 
bending dominated deformation modes, whereas it results in a reduced performance for stretching dominated modes. To explain the dissimilar trends, we first recall that the stretching stiffness of the struts is governed by the ratio $\left(E_{s} A\right) / L$, while the bending stiffness depends on $\left(E_{s} I\right) / L^{3}$. Since for stretching dominated lattices, each macroscopic load is withstood by an axial force, reducing the density always corresponds to a reduction of the crosssection area, and consequently to a decrease in stiffness. On the other hand, bending dominated modes can benefit from the presence of multiple hierarchies, because the density of the strut cross section can be reduced if the material is hierarchically structured. This would penalize, in relative terms, the second moment of area not as much as the area.

We now consider the strength of two-dimensional hierarchical lattices. Since this section focuses on high density lattices $(\lambda>1 / 20)$, we only consider as a failure mode plastic yield, rather than buckling. We remark that the Euler critical stress is given by

$$
\sigma_{c r}=E_{s} \pi \chi^{2} \lambda^{2}
$$

where $\chi=1$ for pinned ends, and $\chi=2$ for fixed ends. Thus for higher density lattices, the Euler critical stress is higher than the yield stress of the material, and the lattice failure is governed by yielding. To obtain the strength of a hierarchical lattice, we must first determine the location and magnitude of stress concentration which emerges at each hierarchical level. Figure 6 shows the stress distribution in response to a unitary macroscopic shear stress applied at the component level.

Similar results were also obtained for other stress states. To obtain the data plotted in Figure 6, the macroscopic stiffness of the lattice has been first determined at each hierarchical level; then a unitary pure shear macroscopic load has been applied at the third hierarchical level and the macroscopic stress distribution, shown in the first column of the plots in Figure 6, has been obtained. Here, the element with the highest effective stress has been identified (circles in the plots) and the component of its strain state has been used as boundary condition to determine the stress distribution in the lattice at level 2. With an equivalent procedure, we calculate the stress of the lattice material at level 1 . We note that at each level the stiffness of the lattice depends on both the cell topology at that level and the macroscopic stiffness of the lattice at the lower level of the hierarchy. In addition, the microscopic stress applied at a location of the lattice in a given level is controlled by the macroscopic stress acting on the lattice at the upper level. For this reason, 

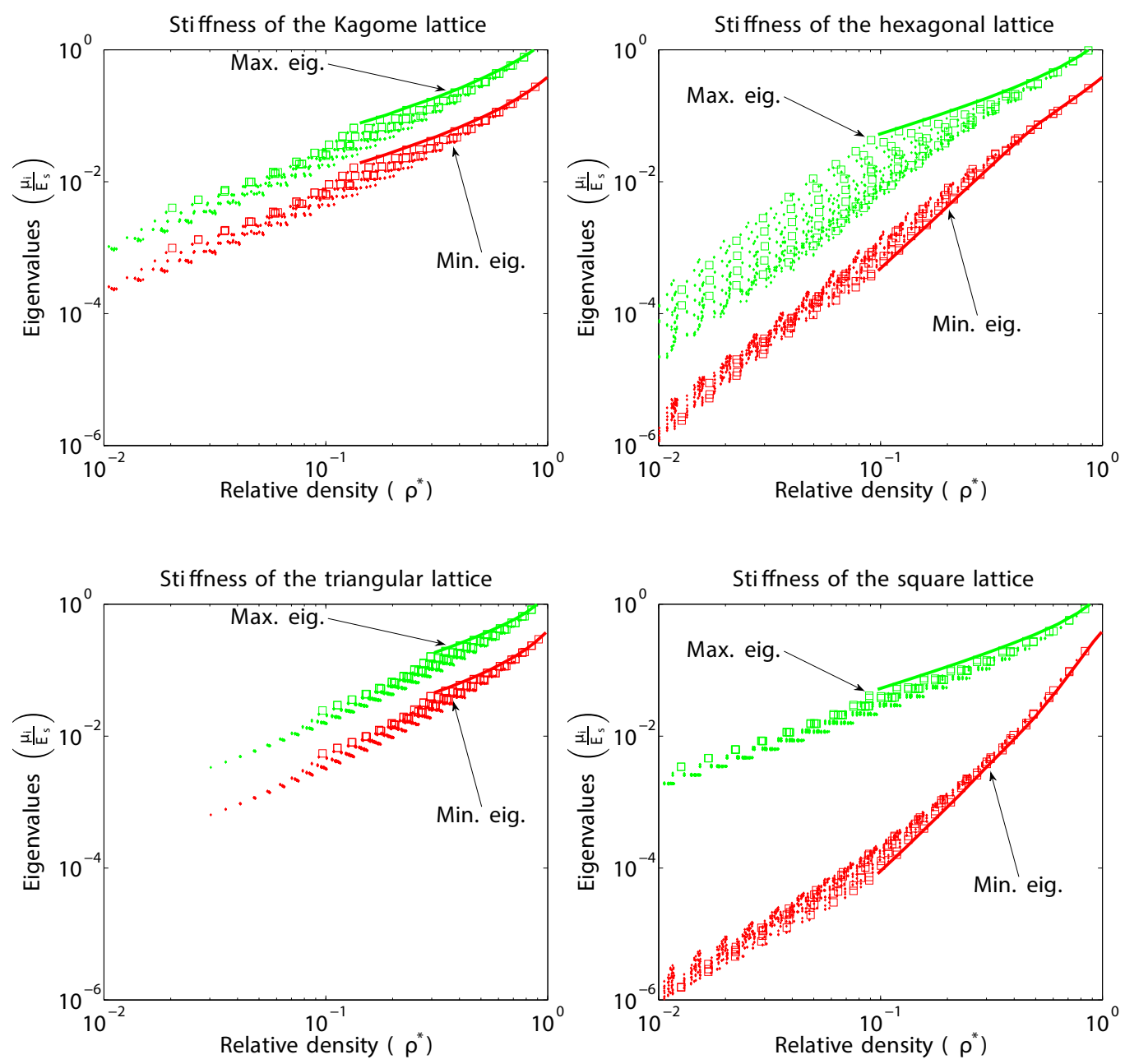

Figure 5: Stiffness of selected high density two-dimensional lattices with three hierarchical levels. A solid line identifies the stiffness of a lattice with one hierarchical level; the empty square markers denote the stiffness of lattices with two hierarchical levels; the solid diamond marker refers to a lattice with three hierarchical levels. The green colour refers to the stiffness for equibiaxial stress, while the red corresponds to the shear stiffness. The Poisson's ratio of the solid material is $\nu=0.3$.

we can conclude that the problem is completely coupled. At each hierarchical level, the stress distribution in the lattice depends on the properties of the 
lattice at the other levels.

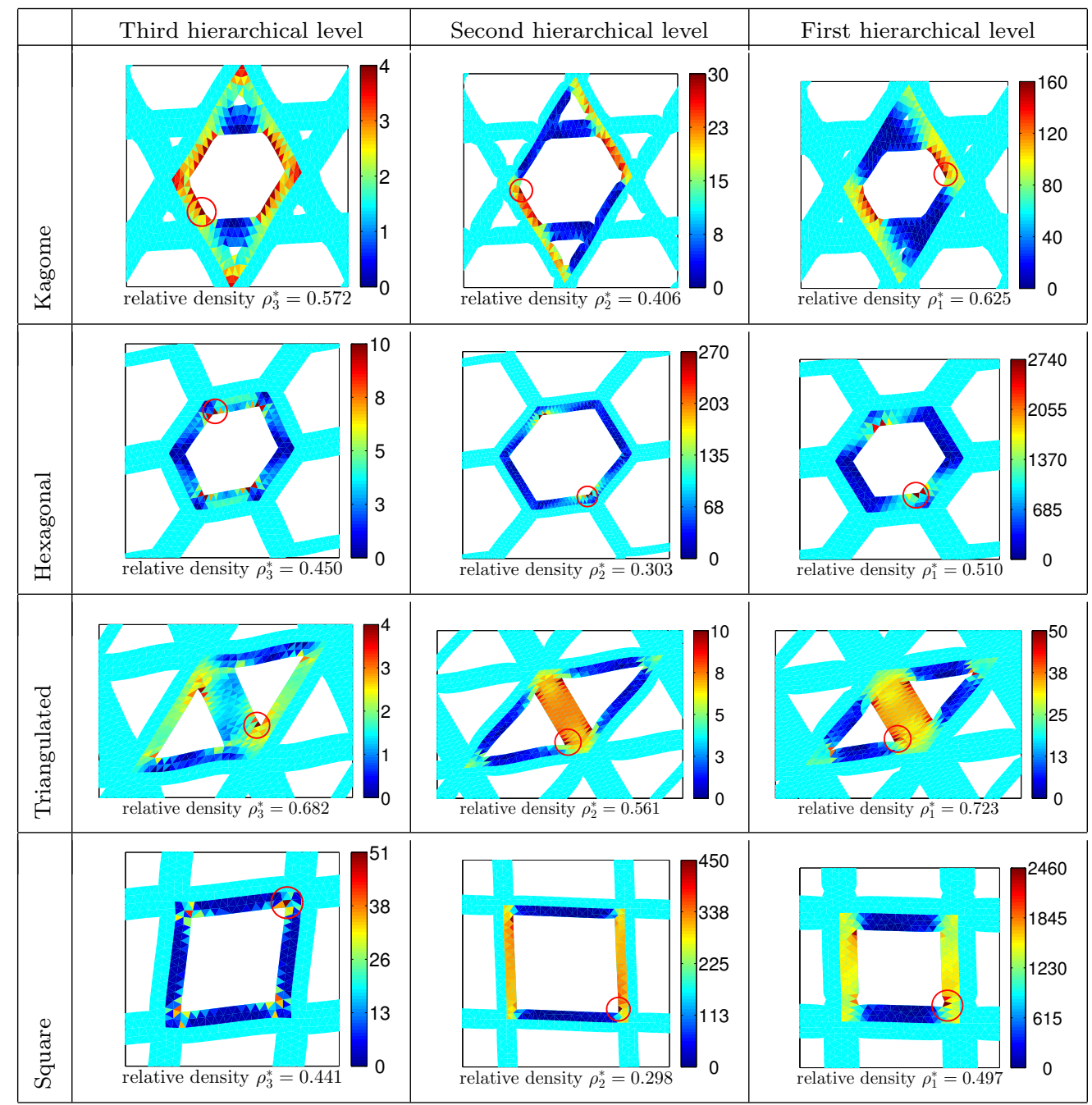

Figure 6: Effective stress amplification factor at each hierarchical level. Colours indicate the dimensionless amplification factor between the applied macroscopic stress and the resulting microscopic stress in the unit cell. At the third hierarchical level, a unitary shear stress is applied, the element with the highest effective stress is identified and the relative stress is applied at the second level. The same procedure is applied at a lower level of the hierarchy to obtain the stress regime.

Figure 7 maps the design space of the yield strength for lattices with 
more than one hierarchical level. In the figure, we plot the inverse of the amplification factor, $\sigma_{e} / \sigma_{e s}$, which represents the load that must be applied at the highest hierarchical level to produce a unitary effective stress in the most stressed location of the solid material. We observe that the existence of multiple hierarchical levels is not beneficial for the lattice topologies under investigation. The points corresponding to lattices with three levels of hierarchy are located below the points corresponding to two hierarchical level lattices, which are below the solid line representing the lattices with one hierarchical level. We also note that the yield strength of the lattices, at each hierarchical level, scales approximately with the first power of the relative density, as expected in a first order approximation. This occurs because for a given macroscopic load, the stress scales with the effective area, over which the load is distributed and which corresponds to the relative density. Yet as shown in Figure 6, material substructuring has the effect of generating uneven stress distribution even if a uniform stress is applied. As a result, high stresses localize in small areas, an effect that is further amplified by structural hierarchy.

\section{Analysis of low density three-dimensional lattices}

Figure 8 shows the three-dimensional topologies examined in this work: the body centred cube (BCC); the regular octet, an optimal truss topology that has been extensively studied in the literature [4, 29]; the cuboctahedron, the only stretching dominated Archimedean polyhedron; and the truncated octahedron, which is the only Archimedean polyhedron, capable of regularly tessellating the space with a unitary packing factor [24]. The truncated octahedron is also of special interest since it is very similar to the tetradecahedron, the polyhedron that minimises the surface to volume ratio. It differs from the tetrakaidecahedron only for a slight curvature of the faces, and approximates the shape under which foams self-arrange [11]. Among the lattices shown in Figure 8, the truncated octahedron is the only bending dominated topology in the open cell configuration, while the others are all stretching dominated in the open cell configuration. All lattices are stretching dominated in their closed cell configuration [27].

For each topology, we calculate the stiffness and the buckling strength of open and closed cell lattices with up to three orders of structural hierarchy. The lattice elements are modelled as beams and shells; hence it is necessary

to enforce the following limits on the slenderness ratio of the edges and of 

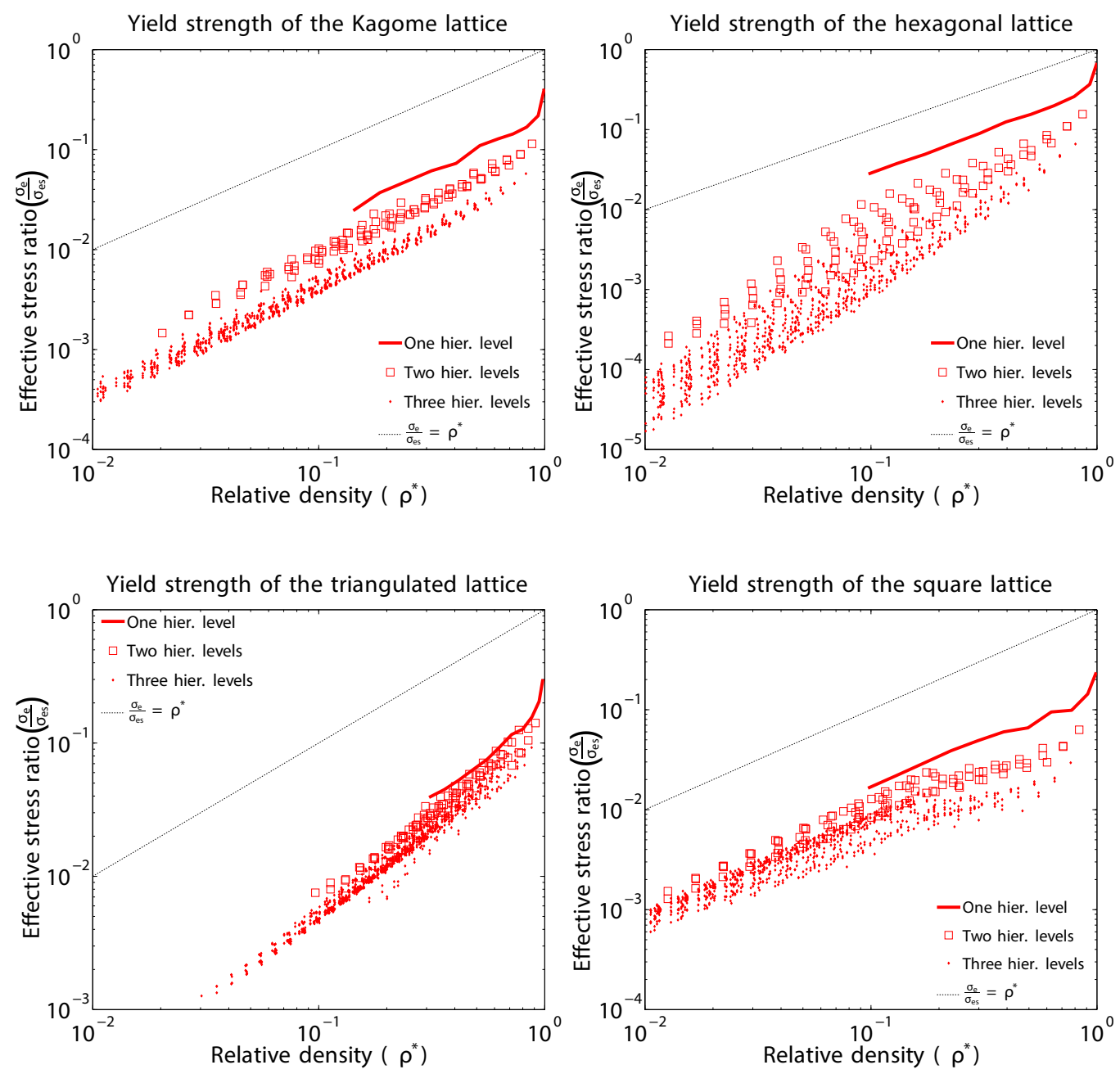

Figure 7: Yield strength of selected high density two-dimensional lattices with three hierarchical levels. A shear macroscopic stress is applied at the third hierarchical level. In contrast to the stiffness (Figure 6), for strength the regions occupied by each hierarchical level are quite distinct; the points relative to higher hierarchical levels are always located below the points representing lower hierarchical levels. Thus, the performance of the lattice decreases with higher levels of hierarchy.

the walls of the lattice:

$$
\frac{L}{d} \leq 20 \quad 0 \leq \frac{t}{d} \leq \frac{1}{2}
$$




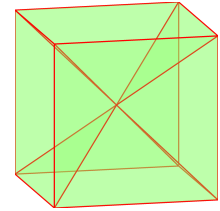

(a) Body Centred Cube (BCC)

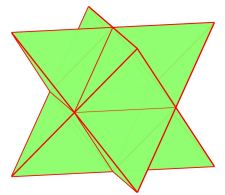

(b) Regular octet

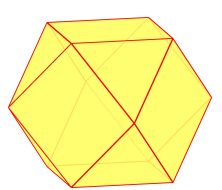

(c) Cuboctahedron

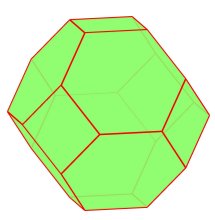

(d) Truncated Octahedron

Figure 8: Three-dimensional topologies under investigation

where $t$ is the thickness of each beam, which we assume have a square cross section, $L$ is the edge length and $d$ is the thickness of the walls. The first inequality guarantees that the Euler-Bernoulli assumption for the beams is fulfilled; the second is to prevent an overestimation of the cell stiffness due to the overlapping portion of adjacent cell elements. To compare the lattice performance for different configurations, we fix the slenderness ratio as $\frac{L}{d}=$ 20 and let $\frac{t}{d}$ vary in the range defined in (3). This choice accounts for the effect of the thickness of the walls on the macroscopic properties of the lattice.

Because of the symmetry of the lattices analysed in this section, there exist nine mutually orthogonal planes of symmetry; hence the stiffness matrix can be written in a reference system with the axes defined by the intersection of the symmetry planes [18] as

$$
\mathbf{K}_{\text {mat }}=\left[\begin{array}{cccccc}
\alpha & \beta & \beta & 0 & 0 & 0 \\
\beta & \alpha & \beta & 0 & 0 & 0 \\
\beta & \beta & \alpha & 0 & 0 & 0 \\
0 & 0 & 0 & \gamma & 0 & 0 \\
0 & 0 & 0 & 0 & \gamma & 0 \\
0 & 0 & 0 & 0 & 0 & \gamma
\end{array}\right]
$$

where the expressions for $\alpha, \beta$ and $\gamma$ depend on the topology and on the properties of the solid material [26]. The eigenvalues and the eigenvectors of $K_{\text {mat }}$ are reported in Table 2. As can be observed, the eigenspace of the stiffness matrix of the lattices examined in this section is similar to that of the lattices analysed in the previous section. In particular, the largest eigenvalue, $\mu_{h}$, corresponds to the hydrostatic macroscopic stress and has single multiplicity. The eigenvalue relative to alternate compression-tension stress, $\mu_{d}$, has both algebraic and geometric multiplicity equal to two and defines deviatoric stress states without shear. The eigenvalue corresponding to shear, $\mu_{s}$, has both algebraic and geometric multiplicity equal to three and corresponds to macroscopic stress states of pure shear. Similarly to the previous section, the three-dimensional lattices show the highest compliance 
for a given shear macroscopic load. Thus in this section we only consider the performance of the lattice for shear stress.

\begin{tabular}{|l|l|l|}
\hline Solid material & Lattice & Eigenvector \\
\hline$\mu_{1 s}=\frac{E_{s}}{1-2 \nu}$ & $\mu_{h}=\alpha+2 \beta$ & $\epsilon_{h}=[1,1,1,0,0,0] \frac{1}{\sqrt{3}}$ \\
\hline$\mu_{2 s}=\frac{E_{s}}{\nu+1}$ & $\mu_{d}=\alpha-\beta$ & $\epsilon_{d 1}=[1,-1,0,0,0,0] \frac{1}{\sqrt{2}}$ \\
& & $\epsilon_{d 2}=[1,0,-1,0,0,0] \frac{1}{\sqrt{2}}$ \\
\hline & & $\epsilon_{s 1}=[0,0,0,1,0,0]$ \\
$\mu_{3 s}=\frac{E_{s}}{2(\nu+1)}$ & $\mu_{s}=\gamma$ & $\epsilon_{s 2}=[0,0,0,0,1,0]$ \\
& & $\epsilon_{s 3}=[0,0,0,0,0,1]$ \\
\hline
\end{tabular}

Table 2: Eigenvalues and eigenvectors of the three-dimensional lattices analysed in the paper. For comparative purposes, the first column reports the eigenvalues of an isotropic homogeneous material.

In Figure 9, the shear stiffness is plotted as a function of the relative density for lattices with one, two and three hierarchical levels. The solid line refers to lattices with one hierarchical level, while the dots refer to lattices with multiple hierarchical levels. The point of the solid line with the smallest relative density refers to the open-cell lattice configuration; for closed-cell lattices, the thickness of the walls increases with the relative density. Figure 9 shows that for low-density lattices each region pertaining to a given hierarchical order is almost disjointed from the others; only a minor overlap exists among them. Since in the closed-cell configuration, all three-dimensional lattices behave as stretching dominated, the lattices with one hierarchical level generally tend to perform better than the lattices with multiple hierarchical orders, as shown by the dots below the solid lines. We also observe that at very low density the shear stiffness of the truncated octahedron tends to rapidly decrease as the bending dominated mode of its open-cell configuration prevails over the stretching dominated behaviour of its close-cell configuration.

Figure 10 shows the strength charts for an applied shear load. Since the focus here is on lattices with low relative density at each level, buckling is considered as the relevant failure mode. The lattice strength is obtained by applying a unitary macroscopic load at the topmost level, the critical loads at each level are calculated, and the smallest critical load is selected as a measure of the global lattice strength. As can be observed in Figure 10, the presence of multiple hierarchical levels has the effect of expanding the 

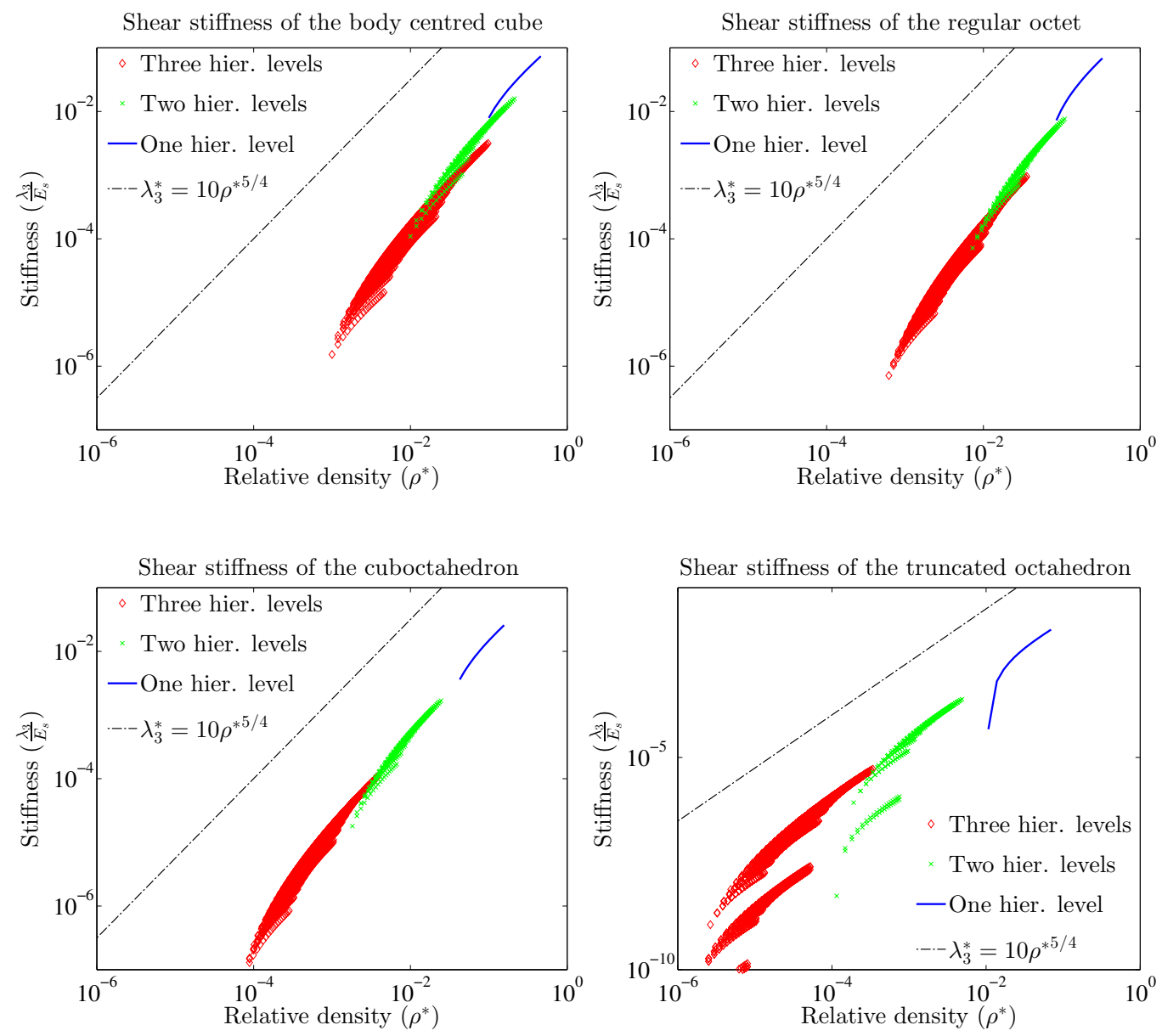

Figure 9: Shear stiffness of three-dimensional topologies. The solid line refers to lattices with a single hierarchical level; the dots to lattice with multiple hierarchical levels.

material properties space. For lattices with a single hierarchical order, the attainable values of the strength are limited to a curve segment that spans one order of magnitude on the abscissa and ordinate axes of the chart. In contrast, the points relative to lattices with two and three hierarchical levels occupy wider regions that span two and three orders of magnitude. 

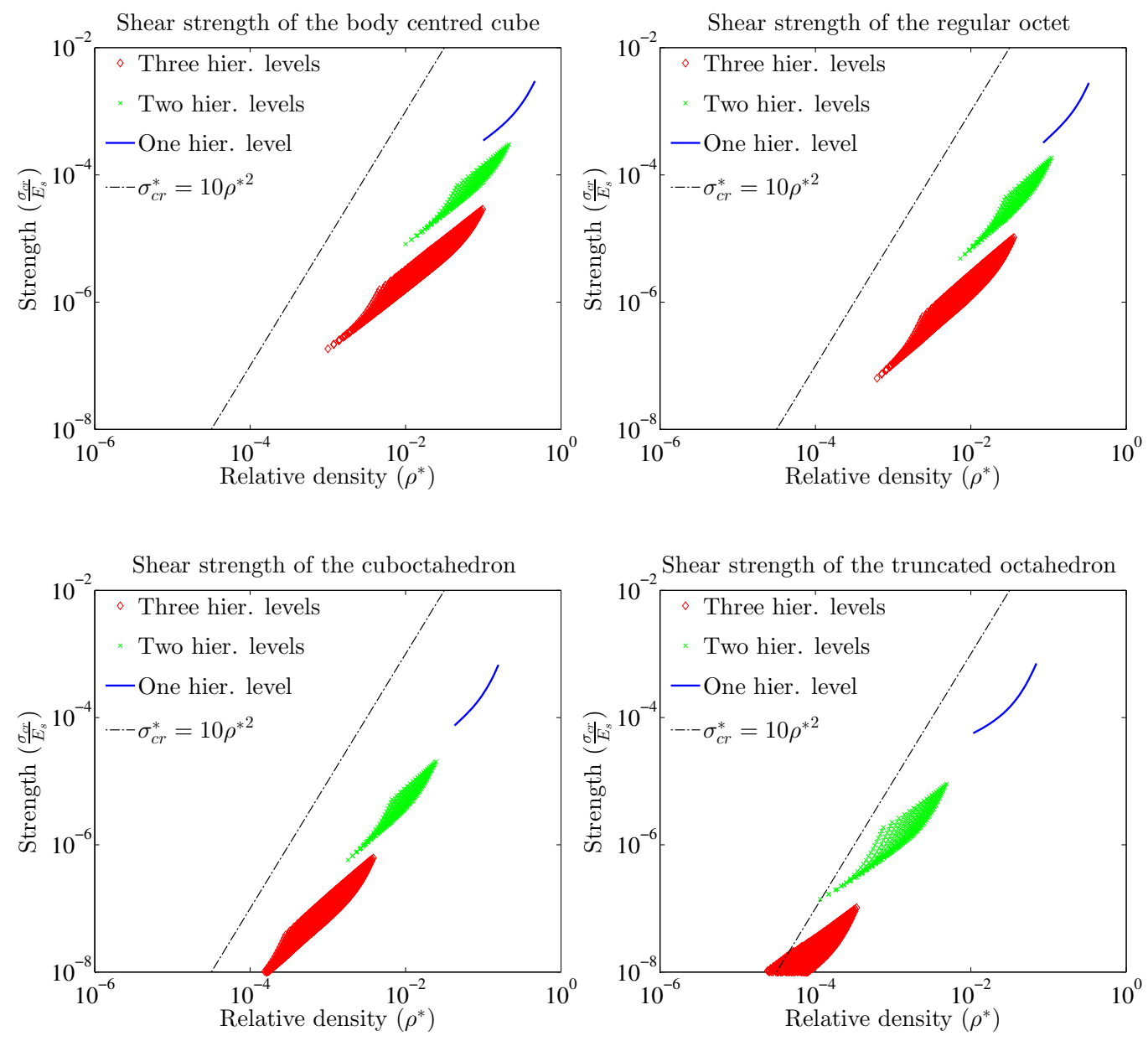

Figure 10: Shear buckling strength for lattices with one, two and three hierarchical levels.

\section{Conclusions}

A scheme based on multiscale mechanics has been applied to determine the stiffness and strength of planar and three-dimensional lattices with multiple orders of structural hierarchy. Four two-dimensional high-density lattices and four three-dimensional low-density lattices have been examined. The analysis has shown the remarkable impact that nesting multiple hierarchical levels has on the stiffness and strength of a hierarchical lattice. The findings provide insight into how the properties evolve with the number of hierarchi- 
cal orders. From the results, it emerges that the property space of a material can be considerably extended without penalizing its specific structural performance and without the need to recur to other materials.

The effect of introducing multiple hierarchical orders is mainly controlled by the lattice topology at each hierarchical level. Bending dominated lattices benefit from a multilevel sub-structuring to a larger extent than the stretching dominated. The presence of micro-voids in bending dominated lattices contributes to reduce the cross-section area without severely penalizing the second moment of area. Thus, the bending stiffness can be preserved efficiently, and increasingly optimised as the relative density decreases. For strength, we have observed that the presence of multiple hierarchical levels in a perfectly periodic lattice produces stress concentrations that reduce the yield strength of the material at the macroscale.

The method presented in this paper can help to guide the design of ultralightweight microlattices, as well as it can be applied to other hierarchical materials, such as nacre and other biological tissues, which do possess a periodic - although not cellular - microarchitecture.

\section{Acknowledgements}

The authors would like to acknowledge the Antje Graupe Pryor Foundation, the McGill International Doctorate Award as well as NSERC (Natural Sciences and Engineering Research Council of Canada) for the support to the research presented in this paper.

\section{Bibliography}

[1] Barthelat, F., 2007. Biomimetics for next generation materials. Philosophical Transactions of the Royal Society a-Mathematical Physical and Engineering Sciences 365 (1861), 2907-2919.

[2] Chen, P. Y., Lin, A. Y. M., Lin, Y. S., Seki, Y., Stokes, A. G., Peyras, J., Olevsky, E. A., Meyers, M. A., McKittrick, J., 2008. Structure and mechanical properties of selected biological materials. Journal of the Mechanical Behavior of Biomedical Materials 1 (3), 208-226.

[3] Deshpande, V. S., Ashby, M. F., Fleck, N. A., 2001. Foam topology bending versus stretching dominated architectures. Acta Materialia 49 (6), 1035-1040. 
[4] Deshpande, V. S., Fleck, N. A., Ashby, M. F., 2001. Effective properties of the octet-truss lattice material. Journal of the Mechanics and Physics of Solids 49 (8), 1747-1769.

[5] Espinosa, H. D., Rim, J. E., Barthelat, F., Buehler, M. J., 2009. Merger of structure and material in nacre and bone - perspectives on de novo biomimetic materials. Progress in Materials Science 54 (8), 1059-1100.

[6] Fleck, N. A., Deshpande, V. S., Ashby, M. F., 2010. Micro-architectured materials: past, present and future. Proceedings of the Royal Society aMathematical Physical and Engineering Sciences 466 (2121), 2495-2516.

[7] Fleck, N. A., Qiu, X. M., 2007. The damage tolerance of elastic-brittle, two-dimensional isotropic lattices. Journal of the Mechanics and Physics of Solids 55 (3), 562-588.

[8] Fratzl, P., Weinkamer, R., 2007. Nature's hierarchical materials. Progress in Materials Science 52 (8), 1263-1334.

[9] Gaytan, S. M., Murr, L. E., Medina, F., Martinez, E., Lopez, M. I., Wicker, R. B., 2009. Advanced metal powder based manufacturing of complex components by electron beam melting. Materials Technology 24 (3), 180-190.

[10] Gibson, L. J., 2012. The hierarchical structure and mechanics of plant materials. Journal of the Royal Society Interface 9 (76), 2749-2766.

[11] Gong, L., Kyriakides, S., Jang, W. Y., 2005. Compressive response of open-cell foams. part i: Morphology and elastic properties. International Journal of Solids and Structures 42 (5-6), 1355-1379.

[12] Koch, K., Bhushan, B., Barthlott, W., 2009. Multifunctional surface structures of plants: An inspiration for biomimetics. Progress in Materials Science 54 (2), 137-178.

[13] Kooistra, G. W., Deshpande, V., Wadley, H. N. G., 2007. Hierarchical corrugated core sandwich panel concepts. Journal of Applied MechanicsTransactions of the Asme 74 (2), 259-268.

[14] Lakes, R., 1993. Materials with structural hierarchy. Nature 361 (6412), $511-515$. 
[15] Meyers, M. A., Chen, P. Y., Lin, A. Y. M., Seki, Y., 2008. Biological materials: Structure and mechanical properties. Progress in Materials Science 53 (1), 1-206.

[16] Parkhouse, J. G., 1984. Structuring: A process of material dilution. In: Third International Conference on Space Structures. pp. 367-374.

[17] Parthasarathy, J., Starly, B., Raman, S., Christensen, A., 2010. Mechanical evaluation of porous titanium (ti6al4v) structures with electron beam melting $(\mathrm{ebm})$. Journal of the Mechanical Behavior of Biomedical Materials 3 (3), 249-259.

[18] Phani, A. S., Fleck, N. A., 2008. Elastic boundary layers in two-dimensional isotropic lattices. Journal of Applied MechanicsTransactions of the Asme 75 (2).

[19] Ramirez, D. A., Murr, L. E., Li, S. J., Tian, Y. X., Martinez, E., Martinez, J. L., Machado, B. I., Gaytan, S. M., Medina, F., Wicker, R. B., 2011. Open-cellular copper structures fabricated by additive manufacturing using electron beam melting. Materials Science and Engineering a-Structural Materials Properties Microstructure and Processing 528 (16-17), 5379-5386.

[20] Rayneau-Kirkhope, D., Mao, Y., Farr, R., 2012. Ultralight fractal structures from hollow tubes. Physical Review Letters 109 (20).

[21] Rho, J. Y., Kuhn-Spearing, L., Zioupos, P., 1998. Mechanical properties and the hierarchical structure of bone. Medical Engineering and Physics $20(2), 92-102$.

[22] Sen, D., Buehler, M., 2011. Structural hierarchies define toughness and defect-tolerance despite simple and mechanically inferior brittle building blocks. Scientific Reports 1.

[23] Stampfl, J., Fouad, H., Seidler, S., Liska, R., Schwager, F., Woesz, A., Fratzl, P., 2004. Fabrication and moulding of cellular materials by rapid prototyping. Int. Jour. of Materials \& Product Technology 21 (4), 285296.

[24] Torquato, S., Jiao, Y., 2009. Dense packings of the platonic and archimedean solids. Nature 460 (7257), 876-U109. 
[25] Torrents, A., Schaedler, T. A., Jacobsen, A. J., Carter, W. B., Valdevit, L., 2012. Characterization of nickel-based microlattice materials with structural hierarchy from the nanometer to the millimeter scale. Acta Materialia 60 (8), 3511-3523.

[26] Vigliotti, A., Pasini, D., 2012. Linear multiscale analysis and finite element validation of stretching and bending dominated lattice materials. Mechanics of Materials 46 (0), 57-68.

[27] Vigliotti, A., Pasini, D., 2012. Stiffness and strength of tridimensional periodic lattices. Computer Methods in Applied Mechanics and Engineering 229, 27-43.

[28] Wadley, H. N. G., 2006. Multifunctional periodic cellular metals. Philosophical Transactions of the Royal Society a-Mathematical Physical and Engineering Sciences 364 (1838), 31-68.

[29] Wallach, J. C., Gibson, L. J., 2001. Mechanical behavior of a threedimensional truss material. International Journal of Solids and Structures 38 (40-41), 7181-7196.

[30] Weiner, S., Wagner, H. D., 1998. The material bone: Structure mechanical function relations. Annual Review of Materials Science 28, 271-298.

[31] Weinkamer, R., Fratzl, P., 2011. Mechanical adaptation of biological materials - the examples of bone and wood. Materials Science \& Engineering C-Materials for Biological Applications 31 (6), 1164-1173.

[32] Yang, S. F., Leong, K. F., Du, Z. H., Chua, C. K., 2002. The design of scaffolds for use in tissue engineering. part ii. rapid prototyping techniques. Tissue Engineering 8 (1), 1-11.

[33] Yao, H. M., Dao, M., Carnelli, D., Tai, K. S., Ortiz, C., 2011. Sizedependent heterogeneity benefits the mechanical performance of bone. Journal of the Mechanics and Physics of Solids 59 (1), 64-74.

[34] Zhao, L., Zheng, Q., Fan, H. L., Jin, F. N., 2012. Hierarchical composite honeycombs. Materials \& Design 40, 124-129. 